\title{
BMJ Open Multicentre, prospective observational study of the correlation between the Glasgow Admission Prediction Score and adverse outcomes
}

\author{
Dominic Jones, ${ }^{\oplus 1}$ Allan Cameron, ${ }^{2}$ David J Lowe, ${ }^{3}$ Suzanne M Mason, ${ }^{1}$ \\ Colin A O'Keeffe, ${ }^{1}$ Eilidh Logan ${ }^{4}$
}

To cite: Jones D, Cameron A, Lowe DJ, et al. Multicentre, prospective observational study of the correlation between the Glasgow Admission Prediction Score and adverse outcomes. BMJ Open 2019;9:e026599. doi:10.1136/ bmjopen-2018-026599

- Prepublication history and additional material for this paper are available online. To view please visit the journal (http:// dx.doi.org/10.1136/bmjopen2018-026599).

Received 14 September 2018 Revised 16 July 2019 Accepted 17 July 2019

Check for updates

(C) Author(s) (or their employer(s)) 2019. Re-use permitted under CC BY-NC. No commercial re-use. See rights and permissions. Published by BMJ.

${ }^{1}$ School of Health and Related Research, University of Sheffield, Sheffield, UK

${ }^{2}$ Acute Medicine, Glasgow Royal Infirmary, Glasgow, UK

${ }^{3}$ Emergency Department, Queen Elizabeth University Hospital

Campus, Glasgow, UK

${ }^{4}$ University of Glasgow School of Life Sciences, Glasgow, UK

Correspondence to

Dr Dominic Jones;

jones.dom17@gmail.com

\section{ABSTRACT}

Objectives To assess whether the Glasgow Admission Prediction Score (GAPS) is correlated with hospital length of stay, 6-month hospital readmission and 6-month allcause mortality. This study represents a 6-month follow-up of patients who were included in an external validation of the GAPS' ability to predict admission at the point of triage. Setting Sampling was conducted between February and May 2016 at two separate emergency departments (EDs) in Sheffield and Glasgow.

Participants Data were collected prospectively at triage for consecutive adult patients who presented to the ED within sampling times. Any patients who avoided formal triage were excluded from the study. In total, 1420 patients were recruited.

Primary outcomes GAPS was calculated following triage and did not influence patient management. Length of hospital stay, hospital readmission and mortality against GAPS were modelled using survival analysis at 6 months. Results Of the 1420 patients recruited, $39.6 \%$ of these patients were initially admitted to hospital. At 6 months, $30.6 \%$ of patients had been readmitted and $5.6 \%$ of patients had died. For those admitted at first presentation, the chance of being discharged fell by $4.3 \%(95 \% \mathrm{Cl} 3.2 \%$ to $5.3 \%$ ) per GAPS point increase. Cox regression indicated a $9.2 \%(95 \% \mathrm{Cl} 7.3 \%$ to $11.1 \%)$ increase in the chance of 6-month hospital readmission per point increase in GAPS. An association between GAPS and 6-month mortality was demonstrated, with a hazard increase of $9.0 \%(95 \% \mathrm{Cl}$ $6.9 \%$ to $11.2 \%$ ) for every point increase in GAPS. Conclusion A higher GAPS is associated with increased hospital length of stay, 6-month hospital readmission and 6-month all-cause mortality. While GAPS's primary application may be to predict admission and support clinical decision making, GAPS may provide valuable insight into inpatient resource allocation and bed planning.

\section{INTRODUCTION}

Crowding gives rise to a myriad of challenges for emergency departments (EDs) and the wider hospital, resulting in poorer clinical outcomes, lower patient satisfaction and an impaired working environment. ${ }^{1-4}$ As demand on EDs and hospitals continues to increase and resources remain limited,
Strengths and limitations of this study

- This is the first study looking at the association between Glasgow Admission Prediction Score (GAPS) and patient outcomes.

- The original derivation of GAPS presents a potential limitation, as it was carried out at a single geographical centre.

- Although this study was conducted at two geographically different regions, both EDs were tertiary units with similar resources.

- Sampling was carried out during a single period at each centre, resulting in possible seasonal idiosyncrasies affecting the results.

- Although it does aid in its implementation, the simplicity of GAPS may limit its accuracy when compared with computerised methods.

data-driven models to ensure operational efficiency will gain increasing importance for improving patient flow. ${ }^{5-9}$

Length of hospital stay (LOS), risk of readmission and mortality are key descriptors of hospital performance. These three factors are all associated with increased costs for healthcare providers. Increasing LOS and hospital readmissions represents risks to patient safety from adverse drug reactions to hospital-acquired infections. ${ }^{10-14}$ Predicting these outcomes at triage could enhance clinical decision making, as well as predicting operational demand, including the need for higher levels of care..$^{91415}$

A clinician assessing a patient in the ED who knows that the patient is probabilistically at a higher risk of mortality, reattendance or prolonged hospital stay may be less inclined to discharge the patient without a more thorough work-up or senior advice and conversely may be less likely to admit a low-risk patient 'just in case' if their clinical parameters put them at a low risk of adverse outcomes. 


\begin{tabular}{|c|c|}
\hline Variable & Points \\
\hline Age & 1 point per decade \\
\hline NEWS* & 1 point per point on NEWS \\
\hline Triage category $\dagger 3$ & 5 \\
\hline 2 & 10 \\
\hline 1 & 20 \\
\hline Referred by a GP & 10 \\
\hline Arrived by ambulance & 5 \\
\hline Admitted <1 year ago & 5 \\
\hline
\end{tabular}

${ }^{*}$ NEWS, National Early Warning Score ${ }^{33}$ (See online supplementary appendix file 1).

†Triage category-Manchester triage system triage category ${ }^{34}$ (See online supplementary appendix 2).

GP, general practitioner.

Moreover, focused and prompt follow-up of patients identified at a high risk of readmission or 6-month mortality could enable a targeted community response. ${ }^{16-20}$

Hospital managers, who need to be able to respond quickly to changes in demand for bed capacity, could have a much clearer idea of predicted bed demand if patients in the ED had an estimated probability of admission and predicted length of stay at an early stage in their visit.

A number of methods and tools such as the HOSPITAL score and LACE index have been shown to be associated with the aforementioned adverse outcomes. ${ }^{21} 22$ However, many are linked to specific patient cohorts and lack the capabilities to predict all of the patient outcomes discussed previously. Most importantly, the majority are not appropriate for use in the ED due to their lack of simplicity and requirement for historical information or information obtained past the point of the ED. ${ }^{101120-27}$

The GAPS (table 1) is a prediction tool, utilising information readily available to predict patient admission at the point of triage in the ED. GAPS was derived and validated from 322000 unselected adult attendances in NHS Greater Glasgow and Clyde. ${ }^{28}$ Furthermore, GAPS has been found to be an accurate predictor of patient disposition and has been found to be superior to triage nurses' ability to predict admission at the point of triage. In addition, GAPS is currently being used at a number of UK sites, including Glasgow, Sheffield, Nottingham and Torbay, to aid in patient streaming in the ED. ${ }^{28-30}$

Although GAPS has been employed as a method of predicting admission, it has not been shown to be associated with adverse patient outcomes, a fact that weakens the case for its widespread adoption. This is the first study looking at the correlation between GAPS and adverse patient outcomes.

\section{METHODS}

This was a prospective observational study aiming to determine whether GAPS is correlated with inpatient length of stay, 6-month hospital readmission and 6-month all-cause mortality. Sampling was carried out at two large EDs in two geographically discrete areas of the UK. This study represents a 6-month follow-up of patients who were included in an external validation of the GAPS' ability to predict admission at the point of triage. The results of this validation are described in an earlier paper. ${ }^{30}$

\section{Setting and participants}

Data were collected on all adult attendances to ED triage at two large teaching hospitals in the UK. They were the Sheffield Teaching Hospitals NHS Foundation Trust ED and the Glasgow Royal Infirmary ED, having approximately 150000 and 95000 annual attendances, respectively.

All patients aged 16 years or below who presented to the ED were not included in the study. Any patients who avoided formal triage by being taken directly to the resuscitation room or to minor injuries were excluded from the study. Finally, patients who left the ED before treatment was complete were also excluded from the analysis.

\section{Sample size}

The power calculation was based on splitting the group into a high GAPS and low GAPS group based on the median GAPS. To have an $80 \%$ probability of demonstrating a hazard ratio (HR) of at least 2 (ie, the high GAPS group having twice the hazard of death of the low GAPS group) with statistical significance $($ at $p<0.05)$ required a minimum of 1307 patients, assuming an overall 6-month mortality of $5 \% .^{31}$ This also meant following patients out to 6 months. Although 30 days would be a more typical time period to assess unplanned reattendance rates, we were able to assess reattendance both at 30 days and at 6 months given the follow-up period.

The sample size needed to demonstrate a similar correlation to both readmission and length of stay would be much smaller than that for mortality because of the much higher event rates. At the sample size to which we were committed by the mortality analysis, there was a near certainty of detecting a HR of 2 for readmission and index length of stay (beta $>0.9999$ )

\section{Data collection}

Sampling was designed to extract data from all time periods equally, totalling 168 hours at each sampling site. Sampling periods were arranged in shifts with researchers collecting required data on all consecutive patients at the point of triage. Data were collected at each site for all consecutive patients who attended during 21 scheduled 8-hour sampling periods. These sampling periods were arranged, so every hour of each day was represented once at each site. At the Sheffield site, data were collected between the 8 and 17 February 2016 and at the Glasgow site, between the 5 and 26 May 2016.

GAPS was then calculated for each patient independent of their clinical management. Any patients admitted to hospital from the ED were followed up to hospital 
Table 2 Demographics of Sheffield and Glasgow patients

\begin{tabular}{|c|c|c|c|}
\hline Variable & Sheffield & Glasgow & Total \\
\hline Total patients & 637 & 787 & 1424 \\
\hline \multicolumn{4}{|l|}{ Sex } \\
\hline Male & 294 & 407 & 701 \\
\hline Female & 343 & 380 & 723 \\
\hline \multicolumn{4}{|l|}{ Age } \\
\hline $10-19$ & 17 & 17 & 34 \\
\hline 20-29 & 119 & 148 & 267 \\
\hline $30-39$ & 60 & 106 & 166 \\
\hline $40-49$ & 85 & 117 & 202 \\
\hline $50-59$ & 97 & 147 & 244 \\
\hline $60-69$ & 62 & 84 & 146 \\
\hline $70-79$ & 84 & 80 & 164 \\
\hline 80-89 & 76 & 79 & 155 \\
\hline $90+$ & 37 & 9 & 46 \\
\hline \multicolumn{4}{|c|}{ Triage category } \\
\hline 1 & 26 & 0 & 26 \\
\hline 2 & 198 & 185 & 383 \\
\hline 3 & 65 & 528 & 593 \\
\hline 4 & 348 & 72 & 420 \\
\hline 5 & 0 & 2 & 2 \\
\hline \multicolumn{4}{|l|}{ NEWS score } \\
\hline 0 & 224 & 223 & 447 \\
\hline 1 & 187 & 239 & 426 \\
\hline 2 & 84 & 116 & 200 \\
\hline 3 & 60 & 75 & 135 \\
\hline 4 & 30 & 53 & 83 \\
\hline $5+$ & 52 & 81 & 133 \\
\hline
\end{tabular}

Arrival by

ambulance

\begin{tabular}{|lrrr|}
\hline Yes & 333 & 344 & 677 \\
\hline No & 304 & 443 & 747 \\
\hline $\begin{array}{l}\text { Final disposition } \\
\text { Admitted }\end{array}$ & 233 & 334 & 567 \\
\hline $\begin{array}{l}\text { Discharged } \\
\text { Readmitted }\end{array}$ & 404 & 453 & 857 \\
\hline Yes & 178 & 257 & \\
\hline No & 459 & 526 & 435 \\
\hline Mortality & & & 985 \\
\hline Yes & 38 & 42 & 80 \\
\hline No & 599 & 741 & 1340 \\
\hline
\end{tabular}

NEWS, National Early Warning Score.

discharge to determine inpatient length of stay. Patients were then followed up at 6 months to collect data on hospital readmission and all-cause mortality. These data were made available using electronic patient records. Any patients who died in the department or were transferred to another hospital were considered to be admitted to hospital for the purpose of the analysis.

\section{Patient and public involvement}

This study used routinely collected clinical data; therefore, no patient or public involvement was required.

\section{Statistical analysis}

All statistical analysis was carried out using R V.3.2.2. ${ }^{32}$ A univariate Cox proportional hazard regression was used to determine the difference in rates of endpoints according to GAPS. The three outcomes tested were as follows:

1. Inpatient length of stay, where discharge counted as the endpoint. Any inpatient deaths during the index presentation or inpatient lengths of stay greater than 6 months were right-censored.

2. Hospital readmission. Here, the exposure to risk of readmission started at discharge from the index presentation (whether from the ED or, if admitted, from hospital). Any patient who was subsequently admitted via an unscheduled reattendance (and not including those who attended ED but were not admitted) was deemed to have reached the endpoint. Patients who reached 6 months of follow-up from the index presentation without being readmitted were right-censored. Deaths that did not occur in hospital were also right-censored. Patients who died during the index admission were not included as they were never exposed to the risk of readmission.

3. All-cause mortality, with all patients surviving beyond 6 months being right-censored.

Kaplan-Meier curves were generated to illustrate the results of the Cox proportional hazards model, with three approximately equal quantiles (high, medium and low GAPS).

\section{RESULTS}

A total of 1487 patients attended for triage during sampling periods, with 686 patients in Sheffield and 801 in Glasgow. Sixty-three patients left the ED before treatment was completed and were therefore excluded. Another four patients who were admitted were lost to follow-up and consequently removed from the sample. Table 2 displays the demographics of the patients included in the analysis.

This resulted in an overall sample of 1420 patients. Of these, $563(39.6 \%)$ were initially admitted. At 6 months, $435(30.6 \%)$ had been readmitted and $80(5.6 \%)$ had died. The median GAPS was 16 (95\% CI 15 to 17$)$. Figure 1 is a flow chart illustrating this.

The Cox proportional hazards analysis of inpatient length of stay demonstrated a HR for reaching the endpoint of hospital discharge of 0.955 (95\% CI 0.945 to 0.965$)$. This can be interpreted as a $4.3 \%$ (95\% CI $3.2 \%$ to $5.3 \%$ ) reduction in the probability of being discharged from hospital at any time for every one-point increase in GAPS. It is perhaps 




Figure 1 Flow chart showing distribution of measured outcomes. Flow chart displaying the measure outcomes of admission, discharge, readmission and mortality.

more illustrative to say that for every 15-point increase in GAPS, the chance of being discharged at any one time decreased by half. Figure 2 displays the Kaplan-Meier curve for inpatient length of stay in each of the three GAPS quantiles. The median length of stay for those admitted in the low GAPS quantile was 1.1 days (95\% CI 0.9 to 1.6 days) compared with 2.0 days (1.6 to 2.3 days) in the middle quantile and 4.6 days ( 3.6 to 5.0 days) in the highest quantile.

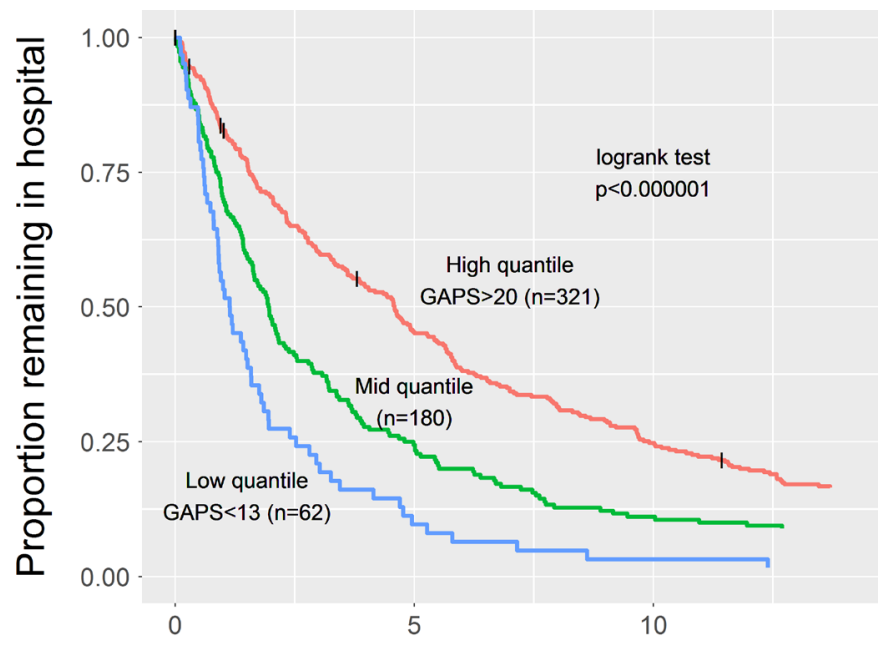

\section{Days after admission}

Figure 2 Kaplan-Meier curve for inpatient length of stay. The data are split into three equal quantiles of low, medium and high GAPS shown by the three separate curves. An increase in GAPS is associated with a longer inpatient length of stay. The logrank test $p$ value indicates that the difference in survival between the quantiles is statistically significant. GAPS, Glasgow Admission Prediction Score.

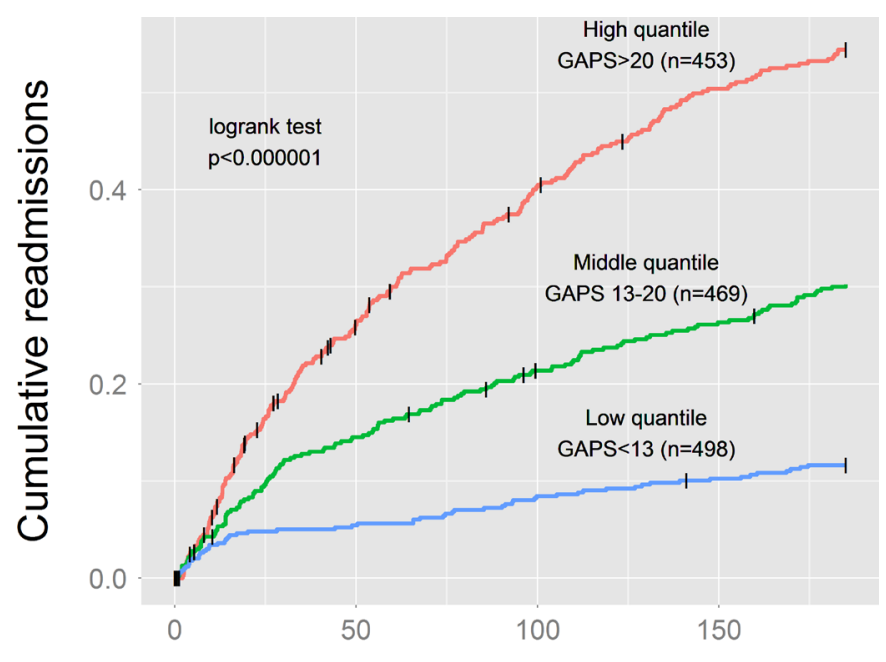

\section{Days after presentation}

Figure 3 Kaplan-Meier curve for 6-month readmission. The data are split into three equal quantiles of low, medium and high GAPS shown by the three separate curves. An increase in GAPS is associated with a higher chance of 6-month hospital readmission. The logrank test $p$ value indicates that the difference in survival between the quantiles is statistically significant. GAPS, Glasgow Admission Prediction Score.

The Cox proportional hazards analysis of 6-month hospital readmission demonstrated a HR of $1.092(95 \%$ CI 1.073 to 1.111). This means that for every one-point increase in GAPS there was a $9.2 \%(95 \%$ CI $7.3 \%$ to $11.1 \%$ ) increase in the risk of hospital readmission at any one time during the 6-month follow-up. This can be represented as saying that for every eight-point increase in GAPS the hazard of hospital readmission doubled. The difference was also statistically significant at 30 days of follow-up, with a HR of 1.048 (1.032 to 1.065). Figure 3 displays the Kaplan-Meier curve for 6-month hospital readmission.

Finally, the Cox proportional hazards analysis of 6-month mortality showed a HR of 1.090 (95\% CI 1.069 to 1.112 ), so that for every one-point increase in GAPS there was a $9.0 \%$ (95\% CI $6.9 \%$ to $11.2 \%)$ increase in the risk of mortality at any one point during the 6-month follow-up. Equivalently, for every eight-point increase in GAPS the risk of mortality doubled. Figure 4 displays the KaplanMeier curve for 6-month mortality.

\section{DISCUSSION}

The results show that higher GAPS, as measured at the point of triage, is associated with increased inpatient length of stay, increased risk of 6-month hospital readmission and increased all-cause mortality, in addition to its established association with increased probability of immediate hospital admission.

These findings suggest that GAPS could be used to help inform clinicians and patients themselves of likely outcomes at an early stage in their hospital visit. GAPS could be utilised to improve flow in the ED, for example, 
by directing low-risk patients to an ambulatory emergency care facility or urgent clinic, by giving junior clinicians a clearer idea of prognosis to support discharge decisions or by directing senior clinicians to the patients to whose care they are most likely to add the most value. ${ }^{28-30}$

Beyond the ED, those patients likely to have a short length of stay could receive early senior input to aid in faster discharges. Higher GAPS could act as a flag for patients who may benefit from more thorough discharge planning, with prompt outpatient follow-up, to mitigate the risks of early readmission.

GAPS may also have a role in indicating hospital bed and other resource usage at an earlier stage. A hospital whose ED can estimate the probability that its patients will be admitted, and how long they are likely to require in hospital, has advance notice of its resource needs.

It could also be utilised on a larger scale, as a way to control for patient differences between departments when measuring hospital performance or to control for differences through time at a single site embarking on service development or performance benchmarking.

The simplicity of GAPS differentiates it from other already available scoring tools associated with patient outcomes. GAPS does not require the use of historical data or aggregation of electronic health records to identify a score, which may be a barrier to adoption. In addition, GAPS can be calculated for both medical and surgical patients. Significantly, it is not a disease-specific tool and could be applied in international health systems. ${ }^{20-27}$

Future research on this topic would involve trialling GAPS in other UK centres outside of Sheffield and Glasgow. Also, further external validation internationally

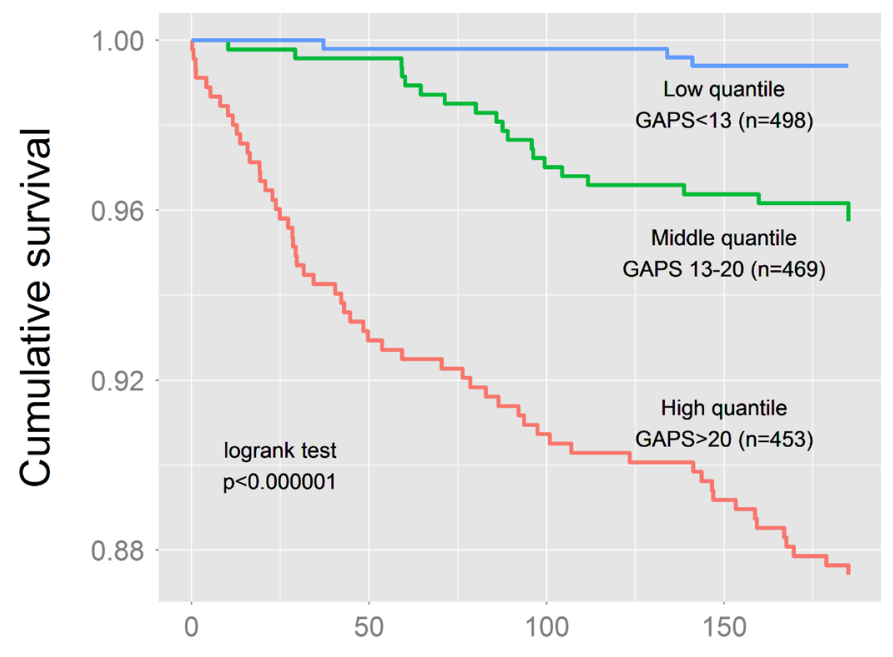

\section{Days after presentation}

Figure 4 Kaplan-Meier curve for 6-month mortality. The data are split into three equal quantiles of low, medium and high GAPS shown by the three separate curves. An increase in GAPS is associated with a higher chance of 6-month mortality. The logrank test $p$ value indicates that the difference in survival between the quantiles is statistically significant. GAPS, Glasgow Admission Prediction Score. would be required to demonstrate widespread applicability. In addition, the practicality of utilising GAPS in real time in an ED and how these insights impact patient flow is yet to be formally evaluated.

This study has a number of limitations that must be highlighted. First, the original derivation of GAPS was carried out at a single geographical centre. Although the current study was conducted at two geographically different regions in the UK, both EDs were tertiary units with similar resources. Sampling was carried out during a single time period at each centre, running the risk of confounding by seasonal variations in attendances and presenting complaints. The simplicity of GAPS may limit its accuracy when compared with complex computerised methods, although the simplicity does help widen its portability. The fact that NEWS and the Manchester triaging system are parameters included in GAPS may limit its application outside of the UK.

Lastly, although this study shows a strong relationship between GAPS and the three outcome measures of interest, the predictive models developed have not been tested prospectively and may vary according to the populations to which they are applied.

\section{CONCLUSION}

This prospective multicentre observational study has shown that higher GAPS are associated with increased inpatient length of stay, increased risk of hospital readmission and increased mortality. These are in addition to previous findings showing GAPS to be an accurate predictor of patient disposition.

Contributors DJ, AC, DJL, SMM, CAOK and EL contributed to the design of the study. EL and DJ collected and recorded the data. DJ wrote the manuscript with significant input from AC, DL, SMM, CAOK and EL during each revision.

Funding Three of the authors of this paper (DJ, CAOK and SMM) were supported by the NIHR Collaboration for Leadership in Applied Health Research and Care Yorkshire and Humber (NIHR CLAHRC YH). www.clahrc-yh.nihr.ac.uk. The views and opinions expressed are those of the author(s) and not necessarily those of the NHS, the NIHR or the Department of Health.

Competing interests None declared.

Patient consent for publication Not required.

Ethics approval The advice of the West of Scotland Research Ethics committee was sought, and it was advised that this study should be considered a service evaluation. Approval was also given by the local Caldicott Guardian in Glasgow and Sheffield.

Provenance and peer review Not commissioned; externally peer reviewed. Data availability statement No data are available.

Open access This is an open access article distributed in accordance with the Creative Commons Attribution Non Commercial (CC BY-NC 4.0) license, which permits others to distribute, remix, adapt, build upon this work non-commercially, and license their derivative works on different terms, provided the original work is properly cited, appropriate credit is given, any changes made indicated, and the use is non-commercial. See: http://creativecommons.org/licenses/by-nc/4.0/.

\section{REFERENCES}

1. Rodi SW, Grau MV, Orsini CM. Evaluation of a fast track unit: alignment of resources and demand results in improved satisfaction 
and decreased length of stay for emergency department patients. Qual Manag Health Care 2006;15:163-70.

2. Sun BC, Hsia RY, Weiss RE, et al. Effect of emergency department crowding on outcomes of admitted patients. Ann Emerg Med 2013;61:605-11.

3. Hoot NR, Aronsky D. Systematic review of emergency department crowding: causes, effects, and solutions. Ann Emerg Med 2008;52:126-36.

4. Guttmann A, Schull MJ, Vermeulen MJ, et al. Association between waiting times and short term mortality and hospital admission after departure from emergency department: population based cohort study from Ontario, Canada. BMJ 2011;342:d2983.

5. National Audit Office. Emergency admissions to hospital: managing the demand. In: Do H, ed. TSO, 2013.

6. Mason S, Mountain G, Turner J, et al. Innovations to reduce demand and crowding in emergency care; a review study. Scand J Trauma Resusc Emerg Med 2014;22:55.

7. Peck JS, Benneyan JC, Nightingale DJ, et al. Predicting emergency department inpatient admissions to improve same-day patient flow. Acad Emerg Med 2012;19:E1045-E1054.

8. Haraden C, Nolan T, Litvak E. Optimizing patient flow: moving patients smoothly through acute care setting: Institute for healthcare improvement 2003.

9. Kelly A-M, Bryant M, Cox L, et al. Improving emergency department efficiency by patient streaming to outcomes-based teams. Aust. Health Review 2007;31:16-21.

10. Donzé J, Aujesky D, Williams D, et al. Potentially avoidable 30-day Hospital readmissions in medical patients: derivation and validation of a prediction model. JAMA Intern Med 2013:173:632-8.

11. Hachesu PR, Ahmadi M, Alizadeh S, et al. Use of data mining techniques to determine and predict length of stay of cardiac patients. Healthc Inform Res 2013;19:121-9.

12. Lucas $\mathrm{R}$, Farley $\mathrm{H}$, Twanmoh J, et al. Emergency department patient flow: the influence of hospital census variables on emergency department length of stay. Acad Emerg Med 2009;16:597-602.

13. Donzé J, Lipsitz S, Bates DW, et al. Causes and patterns of readmissions in patients with common comorbidities: retrospective cohort study. BMJ 2013;347:f7171.

14. Barnes S, Hamrock E, Toerper M, et al. Real-Time prediction of inpatient length of stay for discharge prioritization. J Am Med Inform Assoc 2016;23:e2-10.

15. Lago RJ, Westert GP, Kendrick K. Managing Hospital length of stay reduction: a multihospital approach. Healthcare manage rev 2005;30:82-92.

16. Leppin AL, Gionfriddo MR, Kessler M, et al. Preventing 30-day Hospital readmissions: a systematic review and meta-analysis of randomized trials. JAMA Intern Med 2014;174:1095-107.

17. Dexheimer JW, Leegon J, Aronsky D. Predicting hospital admission at triage in emergency department patients. AMIA Annu Symp Proc $2007 ; 11$.
18. Howell EE, Bessman ES, Rubin HR. Hospitalists and an innovative emergency department admission process. J Gen Intern Med 2004;19:266-8

19. Shadmi E, Flaks-Manov N, Hoshen M, et al. Predicting 30-day readmissions with preadmission electronic health record data. Med Care 2015;53:283-9.

20. Aubert CE, Folly A, Mancinetti M, et al. Prospective validation and adaptation of the hospital score to predict high risk of unplanned readmission of medical patients. Swiss Med Wkly 2016;146:w14355

21. Donzé JD, Williams MV, Robinson EJ, et al. International validity of the hospital score to predict 30-day potentially avoidable Hospital readmissions. JAMA Intern Med 2016;176:496-502.

22. Damery $\mathrm{S}$, Combes $\mathrm{G}$. Evaluating the predictive strength of the lace index in identifying patients at high risk of hospital readmission following an inpatient episode: a retrospective cohort study. BMJ Open 2017;7:e016921.

23. Abhijit S, Harish KV, Radikha N. Particle Swarm optimization over back propagation neural network for length of stay prediction. Procedia computer science 2015;46:268-75.

24. Zhengxing $\mathrm{H}$, Juarez $\mathrm{JM}$, Duan $\mathrm{H}$, et al. Length of stay prediction for clinical treatment process using temporal similarity. Expert systems with applications 2013;40:6330-9.

25. Radovanovic S, Vukicevic M, Kovacevic A. Domain knowledge Based Hierarchical Feature Selection for 30-Day Hospital Readmission Prediction. In: Holmes J, Bellazzi R, Sacchi L, eds. Artificial intelligence in medicine. AIME 2015. Lecture Notes in Computer Science Springer, Cham, 2015.

26. Cotter PE, Bhalla VK, Wallis SJ, et al. Predicting readmissions: poor performance of the lace index in an older UK population. Age Ageing 2012;41:784-9.

27. Cooksley T, Nanayakkara PWB, Nickel $\mathrm{CH}$, et al. Readmissions of medical patients: an external validation of two existing prediction scores. QJM 2016;109:245-8.

28. Cameron A, Rodgers K, Ireland A, et al. A simple tool to predict admission at the time of triage. Emerg Med J 2015;32:174-9.

29. Cameron A, Ireland AJ, McKay GA, et al. Predicting admission at triage: are nurses better than a simple objective score? Emerg Med $J$ 2017;34:2-7.

30. Cameron A, Jones D, Logan E, et al. Comparison of Glasgow admission prediction score and Amb score in predicting need for inpatient care. Emerg Med J (Published online first: 14 February 2018).

31. Schoenfeld DA. Sample-size formula for the proportional-hazards regression model. Biometrics 1983;39:499-503.

32. R Foundation for Statistical Computing. $R$ : a language and environment for statistical computing. Vienna, Austria, 2013.

33. Royal college of physicians. National early warning score with explanatory text: Royal College of physicians, 2012. Available: https://www.rcplondon.ac.uk/projects/outputs/national-earlywarning-score-news [Accessed $24 \mathrm{Feb} 2019]$.

34. Mackway-Jones K, Marsden J, Windle J, et al. Emergency triage: BMJ 2006. 
Correction: Multicentre, prospective observational study of

the correlation between the Glasgow Admission Prediction

\section{Score and adverse outcomes}

Jones D, Cameron A, Lowe DJ, et al. Multicentre, prospective observational study of the correlation between the Glasgow Admission Prediction Score and adverse outcomes. BMJ Open 2019;9:e026599. doi: 10.1136/bmjopen-2018-026599

This article was previously published with incorrect order of authors. The correct order of authors is as follows:

Dominic Jones, Allan Cameron, Suzanne M Mason, Colin A O'Keeffe, Eilidh Logan, David J Lowe

Open access This is an open access article distributed in accordance with the Creative Commons Attribution Non Commercial (CC BY-NC 4.0) license, which permits others to distribute, remix, adapt, build upon this work non-commercially, and license their derivative works on different terms, provided the original work is properly cited, appropriate credit is given, any changes made indicated, and the use is non-commercial. See:http://creativecommons.org/licenses/by-nc/4.0/.

(c) Author(s) (or their employer(s)) 2019. Re-use permitted under CC BY-NC. No commercial re-use. See rights and permissions. Published by BMJ.

BMJ Open 2019;9:e026599corr1. doi:10.1136/bmjopen-2018-026599corr1

Check for updates 